\title{
Multi-Environment Evaluation to Identify Promising Germplasm Lines for Economically Important Traits in G. hirsutum Cotton
}

\author{
Suresh S. Handi* and I.S. Katageri \\ Department of Genetics and Plant Breeding College of Agriculture, University of Agricultural \\ Sciences, Dharwad-580 005, Karnataka, India \\ *Corresponding author
}

\begin{abstract}
Key words
Gossypium hirsutum, Germplasm, Genetic variability, Genetic diversity

Article Info

Accepted:

10 October 2018

Available Online:

10 November 2018 hirsutum check varieties viz., Sahana, Surabhi, MCU 5, DS 28 and ARBH 813 for yield and fibre quality traits. The analysis of variance revealed the presence of significant differences among genotypes and recorded wide range of variations for all the characters over environments. EC296596 (2263 Kg/ha), 128333-Acala-44 (2258 Kg/ha) and $543416 \mathrm{~A} 03 \mathrm{~N} 132(2250 \mathrm{Kg} / \mathrm{ha})$ recorded significantly higher seed cotton yield than superior check, ARBH 813 (1894 Kg/ha). CPD-420, FQT-38, CPD-2011, AKA-8828, DRC-305 and CPD-443 recorded higher ginning outturn ( $>38$ percent) with high boll weight $(>4.0 \mathrm{~g})$, more bolls $(>15.0)$ and more fruiting points $(>35)$. HAG-1055, HLS321729, AK-23B, 543416A03N132, 126663, FQT-38 and 54335402A015 recorded high seed index with ginning outturn ( $>37$ percent), boll weight (4.0g), more bolls $(>14.0)$ and fruiting points $(>35.0)$, moderate height $(>95 \mathrm{~cm}$ to $105 \mathrm{~cm})$. Five germplasm lines viz., FQT-21 (31.8 mm), IC356874 (30.9 mm), ADB-39 (30.9 mm), CPD-437 (30.0 mm) and EC559012 $(30.0 \mathrm{~mm})$ with higher fiber strength $(23.2$ to $24.7 \mathrm{~g} / \mathrm{tex})$ and on par with ARBH 813 for seed cotton yield (1555 to $1966 \mathrm{Kg} / \mathrm{ha}$ ). These germplasm collections represent a valuable resource for improving seed cotton yield and fiber quality in cotton.
\end{abstract}

\section{A B S T R A C T}

Our objective was to evaluate $G$. hirsutum cotton lines, which includes indigenous, exotic collection, released varieties and lines developed from different breeding strategies were evaluated in alpha lattice designs with two replications along with five standard $G$.

\section{Introduction}

Cotton is one of the most important commercial crops which occupied importance from historic days. It is an industrial commodity of worldwide importance.

It occupies the place of pride in Indian agriculture and economy by earning valuable foreign exchange. It is primarily used in textile industries providing highest employment during production, processing, spinning, weaving and marketing throughout the world.

Germplasm is the genetic source material used by plant breeders to develop new cultivars and one of the consequences of successful plant breeding can be increased erosion or reduction in genetic variability for the crop undergoing selection. There is also a danger that valuable genetic resources may be lost to future breeding programs as the areas of genetic 
diversity are developed and as agriculture becomes more intensified (Stoskopf et al., 1993). As a result, breeders need to effectively manage their breeding populations to preserve adequate genetic variation so that future improvements through selection can occur. Proper management of germplasm resources by the breeder includes introducing new germplasm resources on a regular basis to develop new recombinants and hence increase genetic variability. One relevant way to have cotton germplasm information available for breeding purposes is through the evaluation of the existent, yet uncharacterized material. Characters is likely to vary according to the environment in which the genotype is being cultivated, it would therefore, be worthwhile to identify the attributes controlling productivity to suite particular locality.

As mentioned, cotton is natively a crop adapted to tropical and subtropical climates. A shift in the climatic adaptation of the plant has been necessary to enable its successful cultivation in more temperate environments. Germplasm screening for useful characteristics is important for making information available of yet uncharacterized material. The rapid advances in spinning technology in recent decades resulted in increasingly new demands concerning fiber properties. The adoption of new spinning methods, the advances in the traditional spinning process and the higher consumer demands call for extensive research and improvement of the raw material to keep pace with the current quality requirements of the spinning industry. The main objective of this study was to evaluate 320 cotton germplasm lines for both agronomic and fiber characteristics.

\section{Materials and Methods}

In the present study a total of 320 germplasm of $G$. hirsutum lines which includes indigenous, exotic collection, released varieties and lines developed from different breeding strategies with five checks viz., Sahana, Surabhi, MCU 5, DS 28 and ARBH 813 available at Agricultural Research Station, Dharwad Farm were evaluated in alpha lattice designs with two replications. Each replication was subdivided into 16 blocks and each block containing 20 entries representing five environments of two different zones of Karnataka viz. Agricultural Research Station, Dharwad Farm, Institute of organic farming, Dharwad (Zone No. 8) and Agricultural Research Station, Bagalakot (Zone No. 3) under the jurisdiction of University of Agricultural Sciences, Dharwad. Data on seed cotton yield, yield components and fiber quality traits were recorded. The observations on five plants viz., Plant height $(\mathrm{cm})$, No. of monopodia, No. of sympodia, No. of bolls, No. of fruiting points, Boll weight (g), Ginning outturn (\%), Seed index (g), Lint index (g) and Seed cotton yield ( $\mathrm{Kg} / \mathrm{ha})$. The fibre quality parameters included $2.5 \%$ span length, fiber strength, micronaire reading and length uniformity index were analyzed under HVI (high volume instrument) at Central Institute for Research on Cotton Technology (CIRCOT), regional quality evaluation unit situated at ARS, Dharwad farm. The observations on four fiber quality traits of 320 germplasm lines were recorded for two environments (E3-ARS, Dharwad farm and E5- ARS, Bagalkot). Phenotypic data were analyzed using REML procedure (Residual Maximum Likelihood) implemented in Cropstat 7.2 software. The least mean squares obtained after removing the block effects were used in further analysis. Variance components were calculated by fitting a linear mixed model to multi-environment data.The data was subjected to randomized complete block design (RCBD) analysis and analyzed using software, Windostat version 9.1 and frequency distribution curves obtained using SPSS version 16.0 software. 


\section{Results and Discussion}

Germplasm screening for useful characteristics is important for making information available of yet uncharacterized material and has served as a major resource in conjugation with suitable breeding strategies for continuous improvement for yield and fibre quality in cotton. The mean sum of squares for yield traits in $320 \mathrm{G}$. hirsutum cotton germplasm evaluated in five environments is presented in Table 1. The analysis of variance indicated presence of significant variability among the germplasm lines for all yield traits. The data on mean and range for yield traits are presented in Table 2 and 3 represent performance of G. hirsutum check varieties. Frequency distribution of germplasm lines was normal for yield and fibre quality traits (Fig. 1).

Plant height is the important trait in determining the plant architecture suggesting its importance in high density planting and for mechanical harvesting. Wide range of variations for plant height over five environments was recorded. On an average, plant height was highest in PSHEC-15 (121.4 $\mathrm{cm})$ followed by SEC-6 $(118.3 \mathrm{~cm})$, EC479 $(118.0 \mathrm{~cm})$ and 543364A02N46 $(114.8 \mathrm{~cm})$, it was $10 \%$ more height and statistically on par with the tallest check, Surabhi $(103.7 \mathrm{~cm})$ and among the different environments the E5 environment recorded highest plant height $(117.4 \mathrm{~cm})$ with wide range of variation 81.0 $\mathrm{cm}$ to $174.9 \mathrm{~cm}$ respectively. On the contrary the line IC356780 $(71.5 \mathrm{~cm})$ was dwarf than check, MCU $5(86.1 \mathrm{~cm})$ a dwarfest among checks. Tuteja et al., (2006) and Verma and Tuteja (2008) also observed wide range of variations for plant height in cotton.

Monopodium is a main branch of cotton plant present at bottom varying from zero to four. This branch provides support to plant preventing from lodging in intensive method of cultivation with wider row spacing. Plants with higher monopodia are generally robust having more number of sympodia and also having sympodia on monopodia to produce higher yields. In present study the highest number of monopodia was recorded by the line IC357196 (2.89) and is on par with the best check ARBH 813 (2.40). Whereas, in contrast, looking to shortage of labour, identification and utilization of germplasm lines with lower monopodia or zero monopodia to develop variety for mechanical harvesting is necessary. The present study identifies the genotypes with lowest number of monopodia, IC358249 (0.74) fallowed by genotypes IC358249 (0.74), FQT-2 (0.76), GISV-272 (0.77), EC560401 (0.80), EC560392 (0.88), PVK-Rajat (0.89), FQT-35 (0.91), PS-20-2-1 (0.92), EC560399 (0.99) and CPD-448 (0.99), which was significantly lower than check variety Sahana (1.87) over five environments which would resulted in cultivation for high density planting and suitable for machine harvesting and they may be suitable lines in breeding program for less or zero monopodial plants. Krishnadoss and Kadambavanasundaram (1997) have seen 1.7 monopodia branches. Manjula et al., (2004) observed 4.0 monopodia in $G$. herbaceum genotypes. Tuteja et al., (2006) recorded 4.77 monopodia per plant. Nagaraj et al., (2008) noticed 2.15 of monopodia in elite germplasm lines of cotton.

The braches which bear squares and bolls are sympodia and hence directly influence the seed cotton yield. Normally a cotton plant can have around 5 to 25 sympodial branches. In our study, the variability observed for number of sympodia was ranging from 10.3 to 20.5 . Seven germplasm lines have recorded $15 \%$ more sympodia than superior check, ARBH 813 (17.1). In the present study the germplasm line RDT-32 (10.3) followed by 16 germplasm lines recorded lowest number of sympodia and on par with inferior check MCU 5 (13.4) with 
mean of 16.1 over five environments which suggest that they were compact in nature and suitable for dense cropping and machine harvesting. Krishnadoss and Kadambavanasundaram (1997) reported up to 20.9 and 21.0 sympodia in direct and reciprocal cross of tetraploid interspecific crosses. Verma and Tuteja (2008) have seen 65.10 sympodia per plant. Nagaraj et al., (2008) recorded 16.10 sympodia branches. Patel et al., (2009) observed 23-44 sympodia in upland cotton genotypes

The number of bolls is an important trait for the genotype which determines its yielding ability. In the present study, mean value recorded for number of bolls across five environments and all genotypes was 13.7 and only one germplasm line NO-15 (21.2) was recorded higher number of bolls over the superior check ARBH 813 (20.3). Genotypes like CPD-824, ARB-08-822, 126663, NO-4 and RAH-110 recorded more number of bolls (>17) with height more than $>100 \mathrm{~cm}$ and more monopodia (>1.6), sympodia $(>16.0)$. While, the line EC138566 recorded lowest number of bolls which was significantly lesser than inferior check, Sahana (12.6) fallowed by eight germplasm lines recorded less than 10 bolls and their seed cotton yield per hectare were also low $(<1500 \mathrm{Kg} / \mathrm{ha})$. The mean number of bolls of 13.0 (E1), 10.2 (E2), 14.82 (E3), 15.13 (E4), 15.43(E5) with wide range of variation from 2.7 to 21.4 (E1), 0.4 to 31.1 (E2), 7.2 to 26.0 (E3), 7.0 to 23.7 (E4) and 8.3 to 22.8 (E5). About 35.3 bolls per plant were observed by Meena et al., (2006) in upland cotton genotypes. Tuteja et al., (2006) noticed up to 42 bolls per plant. Nagaraj et al., (2008) recorded 16.4 bolls per plant in elite germplasm lines of upland cotton. Baig et al., (2009) noticed 38.07 boll loads per plant.

Fruiting points are the reproductive organs, it depicts potential of plant to bear the flower buds which determines the yielding ability of genotype. Some of these may drop or dry up because of environmental conditions like poor nutrition or pest attack. In present study, wide range of variation was observed for number of fruiting points from 26.0 to 63.3 and two germplasm lines have recorded more than 20 percent fruiting points over superior check, ARBH 813 (50.7). Among these lines RDT-2 recorded highest fruiting points (63.3) but it exhibited poor yield $(1038 \mathrm{Kg} / \mathrm{ha})$ followed by 54335402A015 (62.9) which recorded 14\% higher yield $(2160 \mathrm{Kg} / \mathrm{ha})$ than superior check, ARBH 813 (1894 Kg/ha). Genotypes like CPD-824, ARB-08-822, 126663, CA-105, HAGH-148, HBS-148 and CPD-437 have recorded more number of bolls, fruiting points with more monopoda and sympodia.

Whereas, EC560391 (26.0) fallowed by 21 germplasm lines have recorded lower number of fruiting points than inferior check, Surabhi (29.9). The range of variation observed for fruiting points was 13.8 to $96.3,15.8$ to 98.3 , 20.8 to $54.1,16.5$ to $47.7,29.2$ to 67.4 with the mean values $40.2,39.2,36.5,27.9,48.1$ in E1, E2, E3, E4 and E5 environments.

The lines with big bolls are preferred because of ease in hand picking. It also helps in reducing cost and time involved in manual harvesting. In the present study, over five environments, highest boll weight was recorded by two germplasm lines CPD-431 $(5.23 \mathrm{~g})$ and IC356874 (5.13g) followed by 23 germplasm lines recorded more than 15 percent and 10 percent high boll weight over the superior check, DS $28 \quad(4.45 \mathrm{~g})$ respectively. CPD-431 and IC356874 recorded 43.8 percent and 19.1 percent higher seed cotton yield than check DS 28 (1305 $\mathrm{Kg} / \mathrm{ha}$ ) respectively. However, the line RS810-SGNR (3.12g) recorded significantly lower boll weight than Surabhi (3.78g), an inferior check. The mean value of $4.35 \mathrm{~g}(\mathrm{E} 1)$, 3.75g (E2), 4.40g (E3), 4.11g (E4) and 4.52g (E5) exhibited by this trait with wide range of 
variation from $2.98 \mathrm{~g}$ to $6.74 \mathrm{~g}$ (E1), $2.58 \mathrm{~g}$ to $5.05 \mathrm{~g}$ (E2), $2.80 \mathrm{~g}$ to $6.10 \mathrm{~g}$ (E3), $2.73 \mathrm{~g}$ to $5.79 \mathrm{~g}$ (E4), and $3.15 \mathrm{~g}$ to $6.43 \mathrm{~g}$ (E5). Palomo and Davis (1983) observed $6.46 \mathrm{~g}$ boll weight in interspecific $F_{1} s$ of tetraploid cottons. Meena et al., (2006) observed $3.8 \mathrm{~g}$ boll weight in upland cotton germplasm lines. Tuteja et al., (2006) recorded $3.32 \mathrm{~g}$ boll weight.

Ginning outturn depicts the potential of genotype to yield lint which is raw material for textile industry. Hence genotype with high ginning outturn is considered to be good. The lines with high ginning outturn and high seed cotton yield are preferred by breeder in order to maintain interest of farmers and textile industry (ginning factories). Four germplasm lines viz., CPD-460 (40.23 percent), NH-2211 (39.92 percent), EC560430 (39.84 percent) and CPD-423 (39.53 percent) recorded more than 5.0 percent higher ginning outrun with 24.44 percent $(1624 \mathrm{Kg} / \mathrm{ha}), 10.88$ percent $(1447 \mathrm{Kg} / \mathrm{ha}), 36.7$ percent $(1784 \mathrm{Kg} / \mathrm{ha})$ and 26.05 percent $(1645 \mathrm{Kg} / \mathrm{ha})$ higher yield than DS 28 (37.59 percent ginning outturn and $1305 \mathrm{Kg} / \mathrm{ha}$ seed cotton yield) respectively.

CPD-420, FQT-38, CPD-2011, AKA-8828, DRC-305 and CPD-443 have recorded higher ginning outturn ( $>38$ percent), with high boll weight $(>4.0 \mathrm{~g})$, more bolls $(>15.0)$ and more fruiting points $(>35)$. Whereas, FQT-21 (30.05 percent) recorded significantly lower than inferior check, Surabhi (34.56 percent) over five environments.

The range of variation observed for this trait was from 25.31 to 42.46 percent, 28.50 to 41.04 percent, 29.82 to 40.89 percent, 28.75 to 40.75 percent, and 30.22 to 40.32 percent with the mean value of 36.01 percent, 36.53 percent, 36.76 percent, 35.91 percent, and 36.60 percent and in E1, E2, E3, E4 and E5 respectively. Krishnadoss and Kadambavanasundaram (1997) recorded 30.7 per cent ginning outturn. Tuteja et al., (2006) recorded 34.3 per cent ginning outturn. Verma and Tuteja (2008) have seen 34.75 per cent ginning outturn in genotypes of upland cotton developed using different cytoplasmic sources. Nagaraj et al., (2008) observed 37.14 per cent ginning outturn in elite germplasm lines of upland cotton.

The relationship between seed index and seed cotton yield is complex, as large seeds with more surface area can bear more number of fibers, but at the same time the total available area for number of fiber decreases as seed index increases to high or too low. Hence moderate seed index about 8 to $10 \mathrm{~g}$ is desirable to achieve higher seed cotton yield and ginning outturn.

The variation observed for seed index was from $6.6 \mathrm{~g}$ to $12.15 \mathrm{~g}$. On an average over five environments, the genotype IC356874 $(12.15 \mathrm{~g})$ recorded more than 30.33 percent of seed index which was significantly higher than best check, Sahana (9.33g) followed by eight germplasm lines. Germplasm lines like HAG1055, HLS-321729, AK-23B, 543416A03N132, 126663, FQT-38 and 54335402A015 have recorded high seed index with ginning outturn (>37 percent), boll weight (4.0g), more bolls (>14.0), fruiting points (>35.0) and moderate height $(>95 \mathrm{~cm}$ to $105 \mathrm{~cm})$.

Whereas Tiny-boll (6.60g) showed low seed index and on par with inferior check i.e. ARBH $813(7.59 \mathrm{~g})$. The range of variation exhibited in different environments was 6.35 to $14.35,4.15$ to $11.65 \mathrm{~g}, 6.75$ to $12.5 \mathrm{~g}, 6.25$ to $12.75 \mathrm{~g}, 6.50$ to $12.00 \mathrm{~g}$ with the mean values of $10.73,7.6,9.23,8.63$ and 8.69g in E1, E2, E3, E4 and E5 respectively. Palomo and Davis (1983) reported $14 \mathrm{~g}$ seed index. Meena et al., (2006) recorded $10.8 \mathrm{~g}$ in upland cotton germplasm lines. Bourland and Jones (2009) recorded seed index of $11.1 \mathrm{~g}$. 


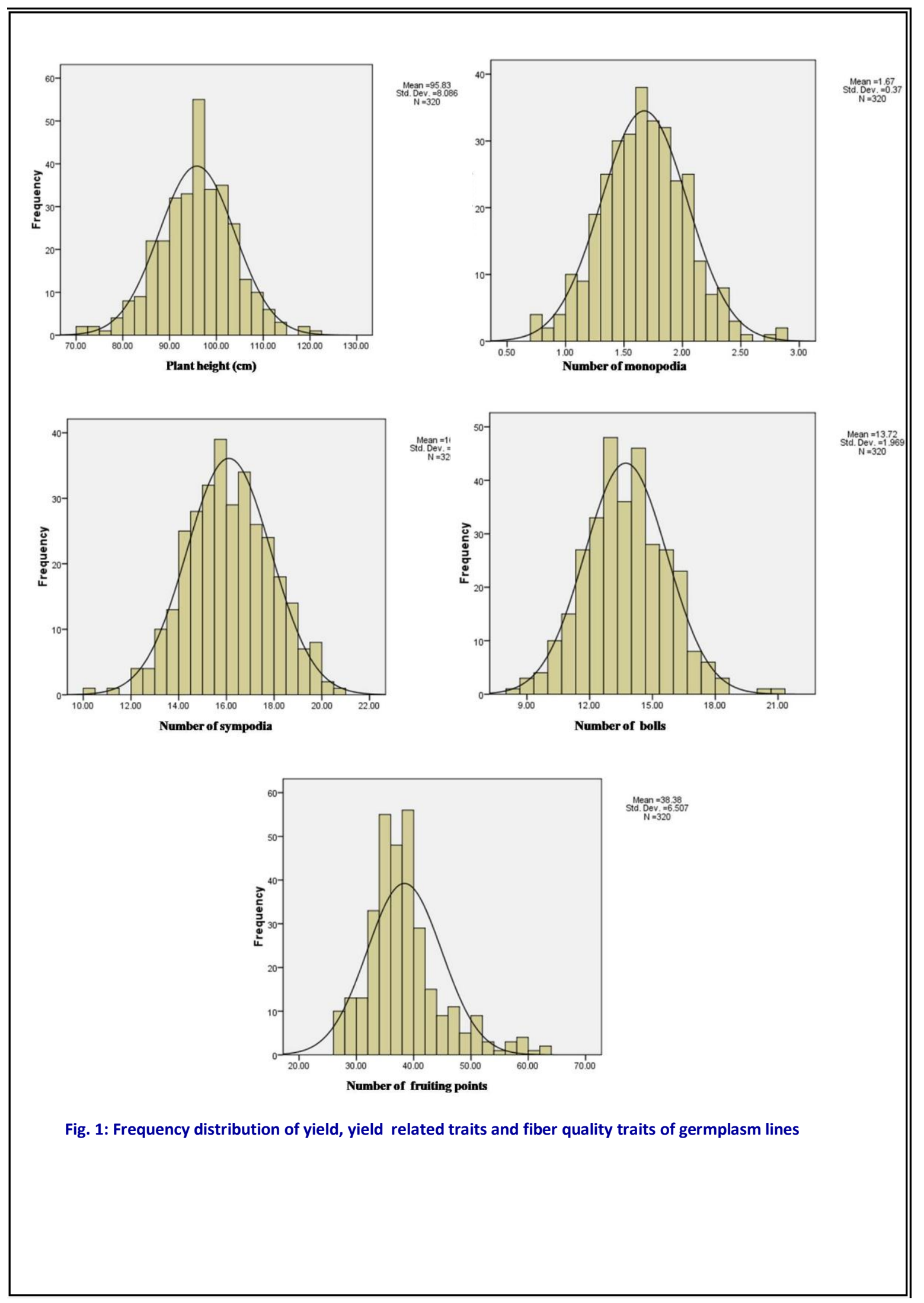


Fig. 1: Contd...
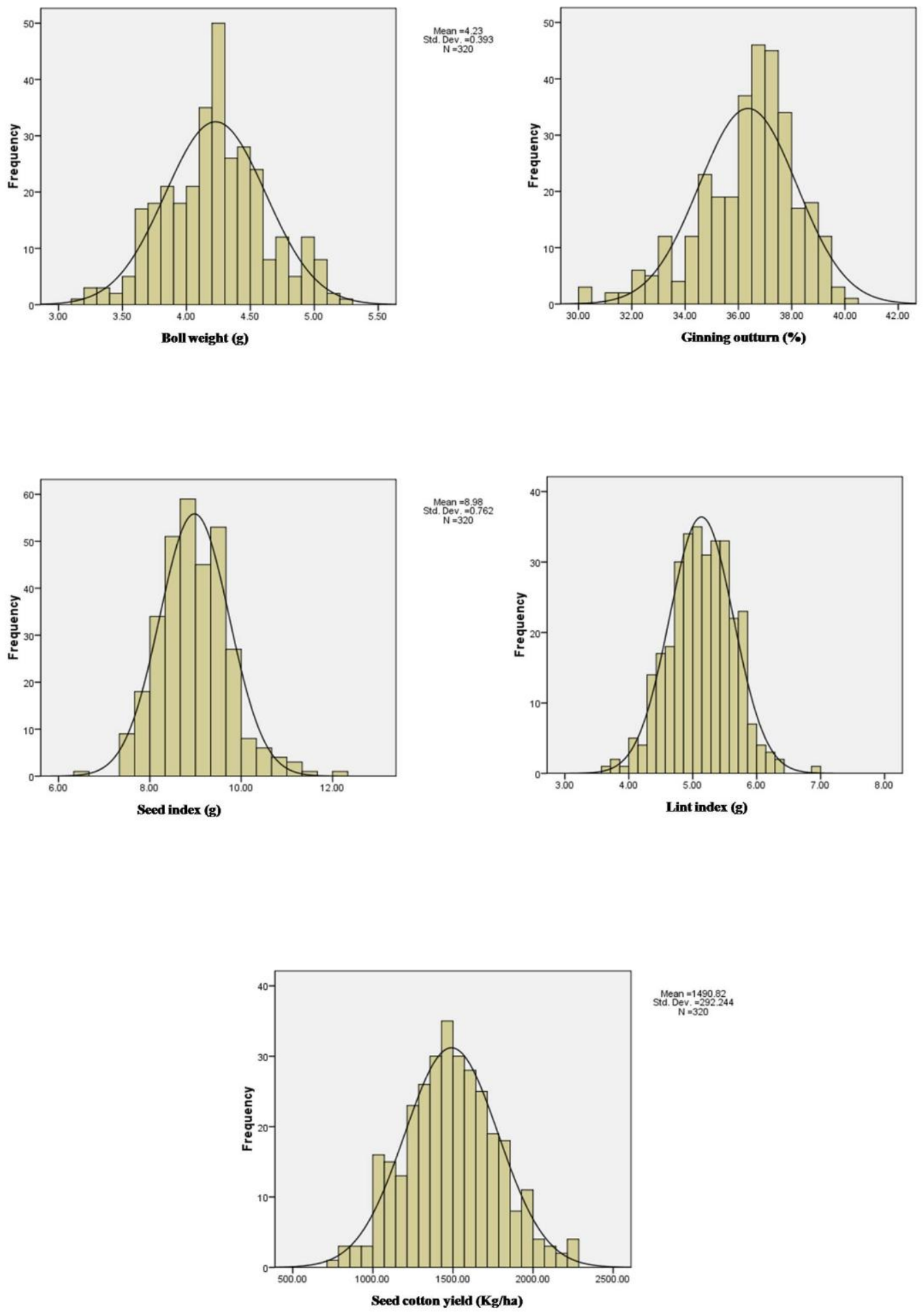


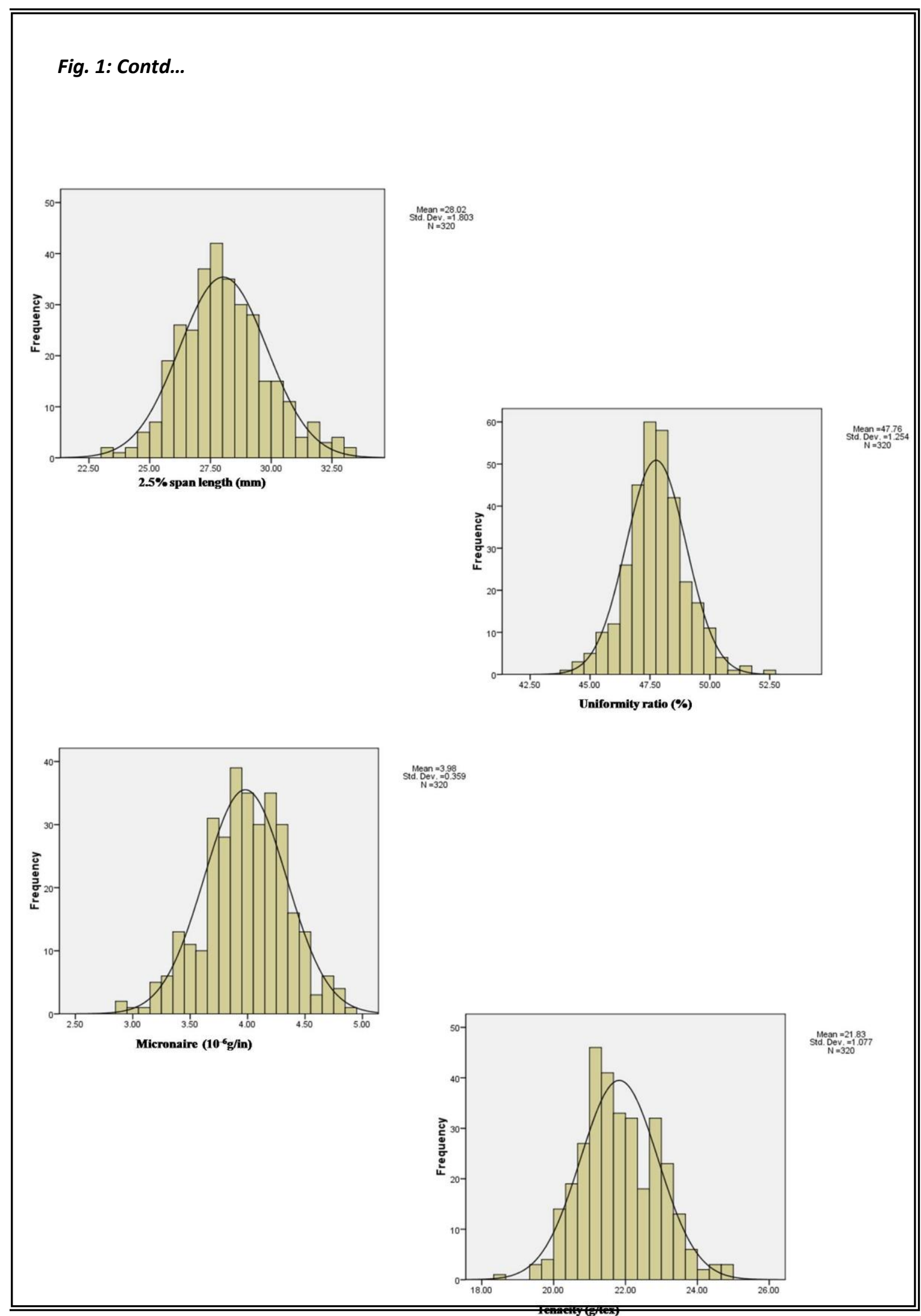


Table.1 Pooled analysis of variance for yield and yield component traits in cotton germplasm lines

\begin{tabular}{|c|c|c|c|c|c|c|c|c|c|c|c|c|}
\hline $\begin{array}{l}\text { Source of } \\
\text { variation }\end{array}$ & Location & Df & $\begin{array}{c}\text { Plant } \\
\text { height } \\
\text { (cm) }\end{array}$ & $\begin{array}{c}\text { No. of } \\
\text { monopodia }\end{array}$ & $\begin{array}{c}\text { No. of } \\
\text { sympodia }\end{array}$ & $\begin{array}{l}\text { No. of } \\
\text { bolls }\end{array}$ & $\begin{array}{l}\text { No. of } \\
\text { fruiting } \\
\text { points }\end{array}$ & $\begin{array}{c}\text { Boll } \\
\text { weight } \\
\text { (g) }\end{array}$ & $\begin{array}{c}\text { Ginning } \\
\text { Outturn } \\
(\%)\end{array}$ & $\begin{array}{l}\text { Seed } \\
\text { index } \\
(g)\end{array}$ & $\begin{array}{c}\text { Lint } \\
\text { index } \\
\text { (g) }\end{array}$ & SCY (Kg/ha) \\
\hline \multirow[t]{5}{*}{ Replications } & E1 & \multirow[t]{5}{*}{1} & $325.3 * *$ & 0.0048 & 7.14 & 0.009 & 152.0 & 0.046 & 2.40 & 0.145 & $0.803^{*}$ & 20976 \\
\hline & $\mathrm{E} 2$ & & $213.2^{*}$ & 0.002 & 5.02 & 0.770 & 192.2 & 0.033 & 23.27 & 0.039 & 0.00001 & $27079 *$ \\
\hline & E3 & & $384.2 *$ & 0.013 & 12.43 & $71.95 * *$ & 16.13 & $0.197 *$ & 8.62 & 0.044 & 0.820 & 175363 \\
\hline & E4 & & 119.8 & 0.205 & 2.22 & 2.55 & 50.12 & 0.175 & 6.02 & 0.791 & $1.135^{*}$ & 36060 \\
\hline & E5 & & 219.3 & 0.157 & 30.27 & 2.37 & 58.68 & 0.246 & 12.60 & 0.308 & 0.274 & 86118 \\
\hline \multirow[t]{6}{*}{ Genotypes } & E1 & \multirow[t]{6}{*}{319} & $499.3 * *$ & $0.4119 * *$ & $22.56 * *$ & $14.70 * *$ & $68.8 * *$ & $0.823 * *$ & $19.45 * *$ & $5.477 * *$ & $2.790 * *$ & $894987 * *$ \\
\hline & E2 & & $181.8 * *$ & $0.9639 * *$ & $49.05^{* *}$ & $13.40 * *$ & $84.3 * *$ & $0.491 * *$ & $13.98 * *$ & $2.564 * *$ & $1.233 * *$ & $74889 * *$ \\
\hline & E3 & & $255.6 * *$ & $0.638 * *$ & $13.76 * *$ & $23.12 * *$ & $59.19 * *$ & $0.653 * *$ & $9.02 * *$ & $1.862 * *$ & $0.766^{* * *}$ & $734885 * *$ \\
\hline & E4 & & $289.0 * *$ & $0.467 * *$ & $9.78 * *$ & $14.07 * *$ & $57.04 * *$ & $0.576 * *$ & $9.51 * *$ & $2.002 * *$ & $0.774 * *$ & $239649 * *$ \\
\hline & E5 & & $405.5 * *$ & $0.721 * *$ & $22.07 * *$ & $14.70 * *$ & $87.37 * *$ & $0.815 * *$ & $7.85^{* *}$ & $1.629 * *$ & $0.701 * *$ & $355578 * *$ \\
\hline & pooled & & $688.03 * *$ & $1.357 * *$ & $34.07 * *$ & $35.54 * *$ & $250.2 * *$ & $1.136 * *$ & $26.78 * *$ & $4.74 * *$ & $2.11 * *$ & $788812 * *$ \\
\hline \multicolumn{2}{|l|}{ Environment } & 4 & $432930 * *$ & $82.66 * *$ & $23803 * *$ & $\begin{array}{c}3022.5^{*} \\
*\end{array}$ & $\begin{array}{c}33706 * \\
*\end{array}$ & $59.05 * *$ & $90.25 * *$ & $838.1 * *$ & $245.6 * *$ & $252940482 * *$ \\
\hline \multicolumn{2}{|c|}{ G X E interaction } & 1276 & 403.02 & 0.4615 & 20.793 & 18.62 & 176.6 & 0.556 & 8.26 & 2.198 & 1.039 & 377794 \\
\hline \multirow[t]{6}{*}{ Error } & E1 & \multirow[t]{5}{*}{319} & 162.7 & 0.0517 & 4.70 & 1.19 & 24.20 & 0.231 & 8.48 & 0.763 & 0.130 & 37793 \\
\hline & $\mathrm{E} 2$ & & 51.14 & 0.0519 & 10.84 & 3.20 & 19.04 & 0.124 & 5.82 & 0.651 & 0.047 & 4211 \\
\hline & E3 & & 82.9 & 0.103 & 3.381 & 5.49 & 18.75 & 0.05 & 2.34 & 0.332 & 0.240 & 85708 \\
\hline & E4 & & 89.6 & 0.065 & 3.342 & 5.78 & 21.33 & 0.042 & 4.05 & 0.264 & 0.261 & 22266 \\
\hline & E5 & & 185.9 & 0.103 & 7.999 & 5.58 & 15.59 & 0.086 & 3.27 & 0.241 & 0.195 & 27823 \\
\hline & Pooled & 1595 & 114.84 & 0.0752 & 6.054 & 4.25 & 42.58 & 0.151 & 2.39 & 0.451 & 0.175 & 35560 \\
\hline
\end{tabular}

$*, * *$ significant at $5 \%$ and $1 \%$ levels respectively.

E1-ARS, Dharwad (2011), E2- ARS, Dharwad (2012), E3 - ARS, Dharwad (2013), E4-MARS, Dharwad (2013) and E5 - ARS, Bagalkot (2013)

ARS- Agricultural research Station, MARS-Main Agricultural Research Station. 
Table.2 Mean and range for different traits in G. hirsutum germplasm lines evaluated in five environments

\begin{tabular}{|c|c|c|c|c|c|c|c|c|c|c|c|}
\hline & Season & $\begin{array}{c}\text { Plant } \\
\text { height } \\
\text { (cm) }\end{array}$ & $\begin{array}{l}\text { Number of } \\
\text { monopodia }\end{array}$ & $\begin{array}{l}\text { Number of } \\
\text { sympodia }\end{array}$ & $\begin{array}{l}\text { Number } \\
\text { of bolls }\end{array}$ & $\begin{array}{c}\text { Number of } \\
\text { fruiting } \\
\text { points }\end{array}$ & $\begin{array}{c}\text { Boll } \\
\text { weight(g) }\end{array}$ & $\begin{array}{c}\text { Ginning } \\
\text { Outturn } \\
(\%)\end{array}$ & $\begin{array}{l}\text { Seed index } \\
\text { (g) }\end{array}$ & $\begin{array}{l}\text { Lint index } \\
\text { (g) }\end{array}$ & $\begin{array}{c}\text { SCY } \\
(\mathbf{K g} / \mathbf{h a})\end{array}$ \\
\hline \multirow[t]{6}{*}{ Mean } & E1 & 94.6 & 1.32 & 12.1 & 13.0 & 40.2 & 4.35 & 36.01 & 10.73 & 6.05 & 1804 \\
\hline & E2 & 72.3 & 1.98 & 26.1 & 10.2 & 39.2 & 3.75 & 36.53 & 7.60 & 4.39 & 556 \\
\hline & E3 & 93.5 & 1.71 & 13.5 & 14.8 & 36.5 & 4.40 & 36.76 & 9.23 & 5.37 & 2250 \\
\hline & E4 & 101.4 & 1.30 & 11.2 & 15.1 & 27.9 & 4.11 & 35.91 & 8.63 & 4.84 & 1312 \\
\hline & E5 & 117.4 & 2.07 & 17.5 & 15.4 & 48.1 & 4.52 & 36.60 & 8.69 & 5.02 & 1532 \\
\hline & Pooled & 95.8 & 1.67 & 16.1 & 13.7 & 38.4 & 4.23 & 36.36 & 8.98 & 5.14 & 1491 \\
\hline \multirow[t]{6}{*}{ Min. } & E1 & 26.3 & 0.17 & 4.8 & 2.7 & 13.8 & 2.98 & 25.31 & 6.35 & 2.21 & 412 \\
\hline & E2 & 41.1 & 0.46 & 8.6 & 0.4 & 15.8 & 2.58 & 28.50 & 4.15 & 2.15 & 255 \\
\hline & E3 & 58.7 & 0.30 & 8.1 & 7.2 & 20.8 & 2.80 & 29.82 & 6.75 & 3.40 & 675 \\
\hline & E4 & 57.4 & 0.20 & 5.2 & 7.0 & 16.5 & 2.73 & 28.75 & 6.25 & 2.97 & 539 \\
\hline & E5 & 81.0 & 0.45 & 11.0 & 8.3 & 29.2 & 3.15 & 30.22 & 6.50 & 3.06 & 614 \\
\hline & Pooled & 71.5 & 0.74 & 10.3 & 8.1 & 26.0 & 3.12 & 30.05 & 6.60 & 3.64 & 770 \\
\hline \multirow[t]{6}{*}{ Max. } & E1 & 142.5 & 3.13 & 21.9 & 21.4 & 96.3 & 6.74 & 42.46 & 14.35 & 10.02 & 3460 \\
\hline & E2 & 104.3 & 4.33 & 41.3 & 31.1 & 98.3 & 5.05 & 41.04 & 11.65 & 7.59 & 1414 \\
\hline & E3 & 131.5 & 3.70 & 21.4 & 26.0 & 54.1 & 6.10 & 40.89 & 12.50 & 7.42 & 3874 \\
\hline & $\mathrm{E} 4$ & 135.8 & 2.95 & 17.6 & 23.7 & 47.7 & 5.79 & 40.75 & 12.75 & 7.26 & 2414 \\
\hline & E5 & 174.9 & 3.80 & 35.1 & 22.8 & 67.4 & 6.43 & 40.32 & 12.00 & 6.74 & 3314 \\
\hline & Pooled & 121.4 & 2.89 & 20.5 & 21.2 & 63.3 & 5.23 & 40.23 & 12.15 & 6.95 & 2263 \\
\hline \multirow[t]{6}{*}{ CD at $95 \%$} & E1 & 25.10 & 0.45 & 4.27 & 2.15 & 9.68 & 0.95 & 5.73 & 1.72 & 0.71 & 382.48 \\
\hline & E2 & 14.07 & 0.45 & 6.48 & 3.52 & 8.58 & 0.69 & 4.75 & 1.59 & 0.43 & 127.67 \\
\hline & E3 & 17.91 & 0.63 & 3.62 & 4.61 & 8.52 & 0.44 & 3.01 & 1.13 & 0.96 & 575.98 \\
\hline & E4 & 18.62 & 0.50 & 3.60 & 4.73 & 9.09 & 0.40 & 3.96 & 1.01 & 1.01 & 293.58 \\
\hline & E5 & 26.82 & 0.63 & 5.56 & 4.65 & 7.77 & 0.58 & 3.56 & 0.97 & 0.87 & 328.17 \\
\hline & Pooled & 20.51 & 0.53 & 4.70 & 3.93 & 8.73 & 0.61 & 4.20 & 1.28 & 0.79 & 341.58 \\
\hline \multirow[t]{6}{*}{ CD at $99 \%$} & E1 & 33.05 & 0.59 & 5.62 & 2.83 & 12.75 & 1.25 & 7.55 & 2.26 & 0.93 & 503.77 \\
\hline & E2 & 18.53 & 0.59 & 8.53 & 4.64 & 11.31 & 0.91 & 6.25 & 2.09 & 0.56 & 168.16 \\
\hline & E3 & 23.59 & 0.83 & 4.76 & 6.07 & 11.22 & 0.58 & 3.96 & 1.49 & 1.27 & 758.64 \\
\hline & $\mathrm{E} 4$ & 24.53 & 0.66 & 4.74 & 6.23 & 11.97 & 0.53 & 5.21 & 1.33 & 1.32 & 386.67 \\
\hline & E5 & 35.33 & 0.83 & 7.33 & 6.12 & 10.23 & 0.76 & 4.69 & 1.27 & 1.14 & 432.24 \\
\hline & Pooled & 27.01 & 0.70 & 6.20 & 5.18 & 11.50 & 0.81 & 5.53 & 1.69 & 1.05 & 449.89 \\
\hline \multirow[t]{6}{*}{$\mathrm{CV}(\%)$} & E1 & 13.48 & 17.23 & 17.92 & 8.39 & 12.24 & 11.05 & 8.09 & 8.14 & 5.96 & 10.78 \\
\hline & E2 & 9.89 & 11.51 & 12.61 & 17.54 & 11.13 & 9.39 & 6.60 & 10.62 & 4.94 & 11.67 \\
\hline & E3 & 9.74 & 18.77 & 13.62 & 15.83 & 11.86 & 5.08 & 4.16 & 6.24 & 9.12 & 13.01 \\
\hline & E4 & 9.34 & 19.61 & 16.32 & 15.92 & 16.55 & 4.99 & 5.60 & 5.95 & 10.56 & 11.37 \\
\hline & E5 & 11.61 & 15.50 & 16.16 & 15.34 & 8.21 & 6.49 & 4.94 & 5.65 & 8.80 & 10.89 \\
\hline & Pooled & 10.81 & 16.52 & 15.33 & 14.60 & 12.00 & 7.40 & 5.88 & 7.32 & 7.87 & 11.54 \\
\hline
\end{tabular}

E1-ARS, Dharwad (2011), E2- ARS, Dharwad (2012), E3 - ARS, Dharwad (2013), E4-MARS, Dharwad (2013) and E5 - ARS, Bagalkot (2013) 
Table.3 Performance of $G$. hirsutum check lines varieties

\begin{tabular}{|c|c|c|c|c|c|c|c|c|c|c|c|}
\hline & Season & $\begin{array}{c}\text { Plant } \\
\text { height }(\mathrm{cm})\end{array}$ & $\begin{array}{l}\text { Number of } \\
\text { monopodia }\end{array}$ & $\begin{array}{l}\text { Number of } \\
\text { sympodia }\end{array}$ & $\begin{array}{l}\text { Number } \\
\text { of bolls }\end{array}$ & $\begin{array}{l}\text { Number of } \\
\text { fruiting } \\
\text { points }\end{array}$ & $\begin{array}{c}\text { Boll } \\
\text { weight(g) }\end{array}$ & $\begin{array}{c}\text { Ginning } \\
\text { Outturn } \\
(\%)\end{array}$ & $\begin{array}{c}\text { Seed } \\
\text { index }(g)\end{array}$ & $\begin{array}{l}\text { Lint index } \\
\text { (g) }\end{array}$ & $\begin{array}{c}\text { SCY } \\
\text { (Kg/ha) }\end{array}$ \\
\hline \multirow[t]{6}{*}{ Sahana } & E1 & 101.0 & 1.76 & 13.1 & 12.4 & 23.9 & 4.19 & 36.13 & 9.54 & 5.43 & 1625 \\
\hline & E2 & 62.2 & 1.81 & 17.2 & 6.1 & 22.4 & 4.00 & 35.00 & 7.35 & 3.97 & 364 \\
\hline & E3 & 103.5 & 1.90 & 13.3 & 16.6 & 37.8 & 4.29 & 38.80 & 10.25 & 5.74 & 2498 \\
\hline & E4 & 112.2 & 1.50 & 15.1 & 14.1 & 29.0 & 3.69 & 36.08 & 9.75 & 5.51 & 1350 \\
\hline & E5 & 121.5 & 2.40 & 16.3 & 13.9 & 40.4 & 4.55 & 37.89 & 9.75 & 5.95 & 2256 \\
\hline & Mean & 100.1 & 1.87 & 15.0 & 12.6 & 30.7 & 4.14 & 36.78 & 9.33 & 5.32 & 1619 \\
\hline \multirow[t]{6}{*}{ Surabi } & E1 & 104.0 & 2.02 & 13.8 & 14.3 & 25.7 & 3.83 & 34.49 & 9.04 & 4.78 & 1708 \\
\hline & E2 & 69.2 & 1.81 & 23.2 & 12.5 & 24.2 & 2.98 & 34.76 & 7.35 & 3.93 & 848 \\
\hline & E3 & 106.2 & 2.10 & 12.3 & 13.8 & 32.0 & 3.94 & 34.73 & 9.75 & 5.19 & 2607 \\
\hline & E4 & 114.9 & 1.70 & 14.1 & 16.1 & 23.2 & 3.48 & 32.98 & 9.25 & 4.55 & 1464 \\
\hline & E5 & 124.2 & 3.05 & 15.3 & 16.1 & 44.6 & 4.68 & 35.83 & 8.75 & 4.89 & 1881 \\
\hline & Mean & 103.7 & 2.14 & 15.7 & 14.6 & 29.9 & 3.78 & 34.56 & 8.83 & 4.67 & 1702 \\
\hline \multirow[t]{6}{*}{ MCU 5} & E1 & 88.1 & 1.94 & 14.2 & 14.2 & 42.0 & 3.94 & 36.62 & 7.68 & 4.49 & 1409 \\
\hline & E2 & 77.7 & 2.06 & 26.3 & 14.8 & 45.0 & 3.44 & 35.41 & 7.05 & 3.88 & 581 \\
\hline & E3 & 79.4 & 1.80 & 8.1 & 12.2 & 28.2 & 4.06 & 38.54 & 8.50 & 5.34 & 1841 \\
\hline & E4 & 88.0 & 1.40 & 7.4 & 14.5 & 19.3 & 3.49 & 36.01 & 8.00 & 4.49 & 1461 \\
\hline & E5 & 97.4 & 2.30 & 11.0 & 14.5 & 40.8 & 4.40 & 34.96 & 7.00 & 3.77 & 1739 \\
\hline & Mean & 86.1 & 1.90 & 13.4 & 14.0 & 35.0 & 3.87 & 36.31 & 7.65 & 4.39 & 1406 \\
\hline \multirow[t]{6}{*}{ DS 28} & E1 & 92.1 & 1.97 & 15.1 & 14.7 & 36.5 & 4.47 & 37.25 & 8.18 & 4.84 & 1288 \\
\hline & E2 & 58.9 & 1.37 & 19.7 & 14.2 & 33.0 & 3.81 & 39.19 & 6.05 & 3.92 & 753 \\
\hline & E3 & 92.8 & 2.20 & 12.2 & 16.1 & 35.3 & 4.90 & 37.63 & 9.50 & 5.73 & 1568 \\
\hline & E4 & 101.4 & 1.80 & 13.0 & 11.1 & 26.4 & 4.11 & 36.95 & 8.75 & 5.13 & 1060 \\
\hline & E5 & 110.8 & 3.20 & 15.1 & 13.4 & 47.9 & 4.98 & 36.93 & 8.25 & 4.84 & 1859 \\
\hline & Mean & 91.2 & 2.11 & 15.0 & 13.9 & 35.8 & 4.45 & 37.59 & 8.15 & 4.89 & 1305 \\
\hline \multirow[t]{6}{*}{ ARBH 813} & E1 & 97.5 & 2.39 & 16.1 & 20.7 & 67.3 & 4.31 & 37.16 & 7.44 & 4.40 & 1867 \\
\hline & E2 & 62.5 & 2.59 & 26.9 & 16.5 & 63.8 & 3.77 & 38.74 & 6.75 & 4.28 & 636 \\
\hline & E3 & 96.8 & 2.30 & 14.3 & 23.9 & 39.6 & 4.79 & 37.65 & 8.25 & 5.01 & 3127 \\
\hline & E4 & 105.5 & 1.90 & 11.1 & 18.9 & 30.8 & 4.27 & 35.27 & 7.75 & 4.25 & 1692 \\
\hline & E5 & 114.8 & 2.80 & 17.3 & 21.2 & 52.2 & 4.48 & 37.86 & 7.75 & 4.73 & 2150 \\
\hline & Mean & 95.4 & 2.40 & 17.1 & 20.3 & 50.7 & 4.32 & 37.33 & 7.59 & 4.53 & 1894 \\
\hline
\end{tabular}

E1-ARS, Dharwad (2011), E2- ARS, Dharwad (2012), E3 - ARS, Dharwad (2013), E4-MARS, Dharwad (2013) and E5 - ARS, Bagalkot (2013) 
Table.4 Pooled analysis of variance for fiber traits in germplasm collection evaluated during 2013 in two environments

\begin{tabular}{|c|c|c|c|c|c|c|}
\hline $\begin{array}{l}\text { Source of } \\
\text { variation }\end{array}$ & Location & df & $\begin{array}{l}2.5 \% \text { Span } \\
\text { length (mm) }\end{array}$ & $\begin{array}{l}\text { Uniformity } \\
\text { ratio }(\%)\end{array}$ & $\begin{array}{c}\text { Micronaire } \\
\text { (g/in) }\end{array}$ & $\begin{array}{c}\text { Tenacity } \\
\text { (g/tex) }\end{array}$ \\
\hline \multirow[t]{2}{*}{ Repliaction } & E3 & 1 & 0.0015 & 0.225 & $0 . .007$ & 0.324 \\
\hline & E5 & 1 & 2.50 & 0.126 & 0.018 & 0.306 \\
\hline \multirow[t]{3}{*}{ Genotypes } & E3 & \multirow[t]{3}{*}{319} & $9.29 * *$ & $4.926 * *$ & $0.41 * *$ & $4.261 * *$ \\
\hline & E5 & & $7.584 * *$ & $4.536 * *$ & $0.326 * *$ & $3.219 * *$ \\
\hline & Pooled & & $13.01 * *$ & $6.288 * *$ & $0.517 *$ & $4.729 * *$ \\
\hline \multicolumn{2}{|l|}{ Environment } & 1 & $323.08 * *$ & $100.1 * *$ & $1.444 * *$ & $23.05 * *$ \\
\hline \multirow[t]{3}{*}{ Error } & E3 & \multirow[t]{2}{*}{319} & 0.0015 & 1.04 & 0.048 & 1.187 \\
\hline & E5 & & 1.095 & 2.214 & 0.034 & 0.466 \\
\hline & Pooled & & 3.866 & 3.17 & 0.219 & 2.75 \\
\hline
\end{tabular}

E3 - ARS, Dharwad and E5 - ARS, Bagalkot

*, ** significant at $5 \%$ and $1 \%$ levels respectively

Table.5 Mean, range and performance of different $G$. hirsutum check lines for fiber traits evaluated during 2013 at two locations

\begin{tabular}{|c|c|c|c|c|c|}
\hline & Locations & $2.5 \%$ Span length (mm) & $\begin{array}{l}\text { Uniformity ratio } \\
\qquad(\%)\end{array}$ & Micronaire (g/in) & Tenacity (g/tex) \\
\hline \multirow[t]{3}{*}{ Mean } & E3 & 28.5 & 47.5 & 3.97 & 21.98 \\
\hline & E5 & 27.5 & 48.0 & 3.99 & 21.68 \\
\hline & Mean & 28.0 & 47.8 & 3.98 & 21.83 \\
\hline \multirow[t]{3}{*}{ Min } & E3 & 22.5 & 40.0 & 2.20 & 18.40 \\
\hline & E5 & 22.8 & 44.0 & 2.90 & 18.50 \\
\hline & Mean & 23.2 & 44.0 & 2.85 & 18.45 \\
\hline \multirow[t]{3}{*}{$\operatorname{Max}$} & E3 & 36.3 & 53.0 & 5.10 & 26.00 \\
\hline & E5 & 33.4 & 52.0 & 5.20 & 26.80 \\
\hline & Mean & 33.2 & 52.5 & 4.85 & 24.85 \\
\hline \multirow[t]{3}{*}{ Sahana } & E3 & 29.5 & 47.0 & 3.30 & 21.10 \\
\hline & E5 & 29.3 & 47.0 & 3.50 & 22.10 \\
\hline & Mean & 29.4 & 47.0 & 3.40 & 21.60 \\
\hline \multirow[t]{3}{*}{ Surabhi } & E3 & 30.3 & 48.0 & 3.60 & 24.30 \\
\hline & E5 & 27.5 & 49.0 & 3.90 & 22.80 \\
\hline & Mean & 28.9 & 48.5 & 3.75 & 23.55 \\
\hline \multirow[t]{3}{*}{ MCU 5} & E3 & 26.8 & 49.0 & 4.20 & 22.60 \\
\hline & E5 & 22.8 & 51.0 & 5.20 & 19.90 \\
\hline & Mean & 24.8 & 50.0 & 4.70 & 21.25 \\
\hline \multirow[t]{3}{*}{ DS 28} & E3 & 30.3 & 47.0 & 3.90 & 23.50 \\
\hline & E5 & 29.1 & 48.0 & 4.50 & 22.40 \\
\hline & Mean & 29.7 & 47.5 & 4.20 & 22.95 \\
\hline \multirow[t]{3}{*}{ ARBH 813} & E3 & 28.5 & 48.0 & 4.30 & 22.40 \\
\hline & E5 & 28.3 & 47.0 & 4.70 & 21.80 \\
\hline & Mean & 28.4 & 47.5 & 4.50 & 22.10 \\
\hline \multirow[t]{3}{*}{ CD@ $5 \%$} & E3 & 0.08 & 2.01 & 0.432 & 2.14 \\
\hline & E5 & 2.06 & 2.93 & 0.366 & 1.34 \\
\hline & Mean & 3.87 & 3.50 & 0.921 & 3.26 \\
\hline \multirow[t]{2}{*}{$\mathbf{C V}$} & E3 & 0.14 & 2.14 & 5.60 & 4.97 \\
\hline & E5 & 3.80 & 3.10 & 4.66 & 3.15 \\
\hline
\end{tabular}

E3 - ARS, Dharwad (2013) and E5 - ARS, Bagalkot (2013) 
It is also measure of lint yielding ability of a genotype which is used as raw material for textile industry. In present study, the variation recorded for lint index was from $3.64 \mathrm{~g}$ to $6.95 \mathrm{~g}$. Three germplasm lines recorded 20 percent higher lint index than superior check, Sahana (5.32g). The genotypes like CPD-476, CPD-423, CPD-420, CPD-921, Abadhita and ARB-760 recorded high lint index $(>6.0 \mathrm{~g})$ with high ginning outturn (>39.0 percent) and seed index $(>9.3 \mathrm{~g})$, whereas the germplasm lines EC560395, EC137592, ACP-71, JBWR23, IC356874 and L-761 exhibited high seed index $(10.0 \mathrm{~g})$, with high lint index $(>5.5 \mathrm{~g})$ and low ginning outturn ( $<35$ percent). The genotypes like FQT-21, CAK-023A, CSHH198F-Sirsa, 543395A03N98, F-2226, 543370A02N62 have recorded high seed index $(>9.5 \mathrm{~g})$, low lint index $(<4.5 \mathrm{~g})$ and low ginning outturn $(<33$ percent). Whereas, GTHV-08-70 (3.64g) recorded low and statistically on par with inferior check, MCU 5 (4.39g) over five environments. The variation observed for this trait was $2.21 \mathrm{~g}$ to $10.02 \mathrm{~g}, 2.15 \mathrm{~g}$ to $7.59 \mathrm{~g}, 3.40 \mathrm{~g}$ to $7.42 \mathrm{~g}, 2.97 \mathrm{~g}$ to $7.26 \mathrm{~g}$ and $3.06 \mathrm{~g}$ to $6.74 \mathrm{~g}$ with the mean values of $6.05 \mathrm{~g}, 4.39 \mathrm{~g}, 5.37 \mathrm{~g}, 4.84 \mathrm{~g}$ and $5.02 \mathrm{~g}$ in E1, E2, E3, E4 and E5 respectively. Krishnadoss and Kadambavanasundaram (1997) observed 4.3g lint index. Nagaraj et al., (2008) recorded $5.12 \mathrm{~g}$ lint index in elite germplasm lines. Bourland and Jones (2010) registered genotypes with $7.5 \mathrm{~g}$ lint index.

Out of 320 germplasm lines studied, the three germplasm viz., EC296596 (2263 Kg/ha), 128333-Acala-44 (2258 $\mathrm{Kg} / \mathrm{ha})$ and543416A03N132 (2250 Kg/ha) recorded significantly higher yield than high yielding check and six other genotypes although statistically on par but recorded more than 10 percent seed cotton yield than superior check, ARBH 813 (1894 Kg/ha). Therefore nine germplasm lines can be potentially used to identify directly as varieties for commercial cultivation. They possessed desirable level of ginning outturn (35.26 to $38.74 \%$ ), longer fiber length (27.9 to $31.7 \mathrm{~mm}$ except one genotype with $25.4 \mathrm{~mm}$ ) and desirable fiber strength (20.4 to $23.0 \mathrm{~g} /$ tex). 25 germplasm lines were significantly inferior and four germplasm lines recorded 40 percent lower seed cotton yield than inferior check, MCU 5 $(1406 \mathrm{Kg} / \mathrm{ha})$ and among genotypes IC357200 observe lowest yield $770 \mathrm{Kg}$ per. In cotton, important yield contributing traits are number of bolls, boll weight and number of sympodia per plant. The development of superior lines for seed cotton yield depends on seed cotton yield per plant itself or number of bolls, boll weight and number of sympodia per plant. The range of variation was 412 to $3460 \mathrm{Kg}$ per hectare, 255 to $1414 \mathrm{Kg}$ per hectare, 675 to $3874 \mathrm{Kg}$ per hectare, 539 to $2414 \mathrm{Kg}$ per hectare and 614 to $3314 \mathrm{Kg}$ per hectare with mean values 1804, 556, 2250, 1312 and 1532 $\mathrm{Kg}$ per hectare in E1, E2, E3, E4 and E5 environments respectively. Dhamayanthi et al., (2008) observed seed cotton yield of 2301 $\mathrm{kg} / \mathrm{ha}$ while handling the $G$. hirsutum $\mathrm{x} G$. barbadense genotypes. Baig et al., (2009) reported $1121 \mathrm{~kg} / \mathrm{ha}$ in $G$. arboreum genotypes. Patel et al., (2009) recorded maximum of $96.51 \mathrm{~g}$ plant yield in Asiatic cotton lines. Bourland and Jones (2009) observed lint yield of 1265 and $1198 \mathrm{~kg} / \mathrm{ha}$ from Arkot 9623 and Arkot 9625, respectively.

The variability of the fiber properties in cotton is an unfavourable element in a market that pits this natural fiber against artificial, more uniform products represented by synthetic fibers. Fiber properties vary as a function of the cultivar but also as a function of the environment and production practices (Clouvel et al., 1998). The mean sum of squares for fiber quality traits in $320 \mathrm{G}$. hirsutum cotton germplasm evaluated in two environments are presented in Table 4. The 
analysis of variance indicated presence of significant variability among the germplasm lines for all four fiber quality traits. The data on mean and range for yield and fiber traits are presented in Table 5 including checks.

Longer fiber is important in the textile industry because it produces stronger yarn leading to higher-priced end products. Recorded $2.5 \%$ span length was longest in CPD-476 (33.2 mm) which was on par with the superior check, DS 28 (29.7 $\mathrm{mm})$ fallowed by five germplasm lines viz., FQT-21 (31.8 $\mathrm{mm})$, IC356874 (30.9 mm), ADB-39 (30.9 $\mathrm{mm})$, CPD-437 (30.0 $\mathrm{mm})$ and EC559012 $(30.0 \mathrm{~mm})$ with good fiber strength $(23.2$ to $24.7 \mathrm{~g} / \mathrm{tex}$ ) and on par with ARBH 813 for seed cotton yield (1555 to $1966 \mathrm{Kg} / \mathrm{ha})$. Whereas, EC138566 (23.2 $\mathrm{mm})$ recorded lower $2.5 \%$ span length than MCU 5 (24.8 $\mathrm{mm}$ ) with the mean of $28.0 \mathrm{~mm}$ over two environments. In E3 and E5 environments, $2.5 \%$ span length was highest in IC357200 (36.3 mm) and CPD-445 (33.4 mm) whereas, it was lowest in IC359059 $(22.5 \mathrm{~mm})$ and GTHV-08-70 (22.8 $\mathrm{mm})$ with mean of germplasm $28.5 \mathrm{~mm}$ and $27.5 \mathrm{~mm}$ respectively. Varalaxmi has fibre length of $32.7 \mathrm{~mm}$ in upland cotton developed by Katarki (1971). Katageri et al., (2003) recorded $30.0 \mathrm{~mm}$ fibre length in recombinant lines. Nagaraj et al., (2008) observed 27.12 $\mathrm{mm}$ fibre length in 20 elite germplasm lines.

Fiber strength is defined as the force necessary to break the fibers and depends on diameter of the cotton fibers. The average acceptable value of cotton fiber strength is 20 to $24 \mathrm{~g} / \mathrm{tex}$. It directly affects yarn strength. Strong yarn is processed efficiently and easily resulting in high yarn yield. Five genotypes like, FQT-21 (24.7 g/tex), ADB-39 (24.5 g/tex), IC359088 (23.7 g/tex), CPD-824 (23.7 $\mathrm{g} /$ tex) and IC356874 (23.6 g/tex) recorded high fiber strength with long fiber length $(27.4$ to $31.8 \mathrm{~mm})$ and they are also statistically on par with superior check, ARBH 813 for seed cotton yield (1555 to $1866 \mathrm{Kg} / \mathrm{ha})$. Whereas, it was weakest in EC138566 (18.45 g/tex) and on par with inferior check, MCU 5 (21.25 g/tex) over two environments. Mean of germplasm lines for fibre strength was $21.94 \mathrm{~g} /$ tex and $21.68 \mathrm{~g} /$ tex in E3 and E5 environments respectively. Katarki (1971) developed an interspecific hybrid Varalaxmi with $24.8 \mathrm{~g} / \mathrm{tex}$ fibre strength. Kulkarni and Khadi (1998) developed DLSa-17 variety of G. arboreum having fibre strength of 19-21 g/tex. Twenty three recombinant lines were obtained by Katageri et al., (2003) which recorded fibre strength of $22 \mathrm{~g} / \mathrm{tex}$.

Highest uniformity ratio was recorded by CPD-921 (53.0\%, 52.0\% and 52.5\%) than superior check, MCU $5(49.0 \%, 51 \%$ and $50.0 \%$ ) in E3, E5 and over two environments respectively followed by 100 germplasm lines recorded more than 48 percent uniformity ratio, which is considered to be excellent and statistically on par with superior check, MCU $5(50.0 \%)$. However, it was lowest in IC357200 (44.0\%) followed by three lines recorded less than 45 percent uniformity ratio over the check, Sahana $(47.0 \%)$ over two environments that is below average grade for uniformity ratio.48.26\% uniformity ratio was noticed in 20 elite germplasm lines by Nagaraj et al., (2008).

Micronaire is the measure of fiber fineness. Thinner fibers are more durable, brighter and softer. Fine fiber forms stronger yarn and directly affects processing stages. In the present study, five germplasm lines have recorded more than $4.7 \mu \mathrm{g} / \mathrm{in}$ and fifteen lines have recorded fine microniare value lower than Sahana (3.4 $\mu \mathrm{g} / \mathrm{in})$. Among them the line EC170338 (4.9 $\mu \mathrm{g} / \mathrm{in})$ was statistically on par with MCU 5 (4.7 $\mu \mathrm{g} / \mathrm{in})$, whereas it was finest in 543370A02N62 $(2.9 \mu \mathrm{g} / \mathrm{in})$ and on par with check, Sahana (3.4 $\mu \mathrm{g} / \mathrm{in})$ over two 
environments. Mean of germplasm lines for micronaire value was $3.92 \mu \mathrm{g} / \mathrm{in}$ (E3) and $3.99 \mu \mathrm{g} / \mathrm{in}$ (E5). Krishnaswami and Kothandraman (1975) observed lines with 4.0 $\mu \mathrm{g}$ per inch micronaire. Nagaraj et al., (2008) noticed $3.74 \mu \mathrm{g}$ per inch micronaire value in study of 20 elite germplasm lines.

In conclusion the germplasm collections represent a valuable resource with more yield potential along with superior quality which were used for improving cotton varieties with high fibre yield and fiber quality and which are desirable source for the cotton breeding programme in wishes to transfer the desirable traits from the germplasm source to otherwise adapted agricultural cultivars.

\section{References}

Baig, K. S., Chavon, M. H. and Kakde, S. S., 2009, Screening of $G$. arboreum, $G$. hirsutum and introgressed diploid and tetraploid strains derived from interspecific hybridization between cultivated species of cotton for drought tolerance. J. Cotton Res. Dev., 28 (1) 813.

Bourland, F. M. and Jones, D. C., 2010, Registration of Arkot 9811 and Arkot 9815 germplasm lines of cotton. $J$. Plant Regist., 4(3): 232-235.

Clouvel, P., Goze, E., Sequeira, R., Dusserre, J. and Cretener. M., 1998, Variability of Cotton Fiber Quality. p. 963-966. In Proc. World Cotton Research Conference 2, "New Frontiers in Cotton Research" 6-12 Sept. Athens, Greece.

Katageri, I. S., Khadi, B. M. Manohas, K., Soregaon, C. D. Vamadevaiah, H. M. Manjula, S. and Badigannavas, A. M. 2003, Recombinant lines in cotton (Gossypium spp). Papers Presented at World - Cotton Research Conference III, held at Cape Town South Africa. 913 March.
Katarki, B. H., 1971, Varalxmi, A high yielding hybrid cotton of quality. Indian Farming, 21(8): 35-36

Krishnadoss, D. and Kadambavanasundaram, M., 1997, Heterosis in intra and inter specific hybrids in tetraploid cotton. $J$. Indian Soc. Cotton Improv., pp. 110116.

Kulkarni, V. N. and Khadi, B. M., 1998, Long lint $G$. arboreum for meeting textile needs. New Frontiers in Cotton Research: Proceedings of World Cotton Research Conference-Ii, Athens, Grace, Sept. 6-12.

Manjula, S. M., Khadi, B. M., Pawar, S. V., Shobha Immadi and Katageri, I S., 2004, Improvement of $G$. herbaceum through introgression breeding. International Symposium on Strategies for Sustainable Cotton Production - A Global Vision, 1, Crop Improvement, 23-25 Nov, Univ. Agric. Sci., Dharwad, Karnataka (India).

Meena, R., Mongo, A. D., Rajiv Kumar and Hamid Hasan, 2006, Screening of American cotton ( $G$. hirsutum) and Desi cotton ( $G$. arboreum) for fibre quality traits, seed cotton yield and important yield components. Indian J. Plant Genet. Reso., 19(1): 122-124.

Meena, R., Mongo, A. D., Rajiv Kumar and Hamid Hasan, 2006, Screening of American cotton ( $G$. hirsutum) and Desi cotton ( $G$. arboreum) for fibre quality traits, seed cotton yield and important yield components. Indian J. Plant Genet. Reso., 19(1): 122-124.

Nagaraj Basavaraddi, Katageri, I. S., Savita, M. and Khadi, B. M., 2008, Assessment of genetic divergence in elite cotton lines (G. hirsutum L.). J, Indian Soc, Cotton Improv, Dec., 105-112.

Palomo Artura and Davis, D. D., 1983, Response of an $\mathrm{F}_{1}$ interspecific $(G$. hirsutum $\mathrm{x} G$. barbadense) cotton 
hybrid to plant density in narrow rows. Crop Sci., 23: 1053-1056.

Patel, A. I., Mali, S. C., Chhimpi, B. G. and Patel, U. G., 2009, Stability analysis for seed cotton yield and component traits in intra and inter specific crosses of GMS based Asiatic cotton. Research on Crops, 10(3): 655-662.

Stoskopf, N. C., Tomes, D. T. and Christie, B. R., 1993. Plant Breeding, Theory and Practice. Westview Press, Inc. Colorado.
Tuteja, O. P., Sunil Kumar and Mahender Singh, 2006, Selection parameters and yield enhancement of upland cotton $(G$. hirsutum) under irrigated ecosystems of north India. Indian J. Agric. Sci., 76(2): 77-80.

Verma, S. K. and Tuteja, O P., 2008, Assessment of genetic divergence among genotypes of upland cotton $(G$. hirsutum L.) having different cytoplasmic background. J. Indian Soc. Cotton Improv., April, 1-7.

\section{How to cite this article:}

Suresh S. Handi and Katageri, I.S. 2018. Multi-Environment Evaluation to Identify Promising Germplasm Lines for Economically Important Traits in $G$. hirsutum Cotton. Int.J.Curr.Microbiol.App.Sci. 7(11): 929-944. doi: https://doi.org/10.20546/ijcmas.2018.711.109 